\title{
Radiation dose to testes and risk of infertility from radiotherapy for rectal cancer
}

\author{
MICHALIS MAZONAKIS ${ }^{1}$, JOHN DAMILAKIS ${ }^{2}$, HARIS VARVERIS $^{3}$ and NICHOLAS GOURTSOYIANNIS ${ }^{4}$ \\ ${ }^{1}$ Department of Medical Physics, University Hospital of Iraklion, 71110 Iraklion; Departments of ${ }^{2}$ Medical Physics, \\ ${ }^{3}$ Radiotherapy and Oncology, and ${ }^{4}$ Radiology, Faculty of Medicine, University of Crete, 71409 Iraklion, Crete, Greece
}

Received June 3, 2005; Accepted August 24, 2005

\begin{abstract}
This study aims to provide the means for testicular dose estimation from radiotherapy for rectal cancer. Rectal irradiation was simulated on a humanoid phantom using a $6 \mathrm{MV}$ photon beam. The effect of field size, distance from irradiated area, wedge introduction into lateral beams, tissue thickness along the beam axis and use of gonad shields on the testicular dose was examined. Testicular dose was measured in five patients undergoing radiotherapy for rectal carcinoma. For a 4500 cGy tumour dose, testicular dose was 32-216 cGy depending upon the field dimensions and the distance from the field isocenter. The presence of wedges increased the testicular dose by a factor up to 2.2. The increase of irradiated tissue thickness increased the gonadal dose up to $40 \%$ whereas the use of the appropriate gonad shield reduced the dose by $>66 \%$. A simple method was developed to estimate testicular dose. The mean difference between the in vivo gonadal doses and the doses calculated using the proposed method was $5.8 \%$. Testicular dose can exceed the value of $100 \mathrm{cGy}$, which permits a complete recovery of spermatogenesis. The presented data can be used to estimate the gonadal dose and the associated risk of infertility attributable to rectal irradiation.
\end{abstract}

\section{Introduction}

Rectal cancer can occur any time during adulthood (1). Reported experience has suggested that a significant number of young and middle-aged male patients present with carcinoma of the rectum $(2,3)$. One of the most important component of multimodality care employed in these patients is the preoperative or postoperative external beam radiotherapy. However, testicular exposure to ionizing radiation is associated with an increased risk of temporary or permanent infertility depending upon the amount of absorbed dose to gonads (4). Testicular dose anticipation is of great importance because it allows the assessment of the risk for reduced reproductive capacity.

Correspondence to: Dr John Damilakis, Department of Medical Physics, Faculty of Medicine, University of Crete, P.O. Box 1393, 71409 Iraklion, Crete, Greece

E-mail: damilaki@med.uoc.gr

Key words: radiotherapy, rectal cancer, testicular dose, infertility
Only a few reports exist in the literature dealing with the dose to testes during radiotherapy for rectal cancer $(2,3,5,6)$. Budgell et al (2) have measured the total testicular dose from abdominopelvic radiotherapy using a single irradiation technique. The above dosimetric data have not accounted for the contribution of scattered dose to testes from each treatment field separately and, therefore, they can not be applied whenever alterations in beam weight or field arrangement take place. Moreover, the effect of shielding the testes on the gonadal dose was not investigated. Piroth et al (3), Dueland et al (5) and Hollenhorst et al (6) have measured the scattered gonadal doses in vivo using thermo-luminescent dosemeters. Scant information has been presented for factors influencing the dose to testes, i.e. field dimensions, distance separating the testes from treatment field, introduction of beam modifying absorbers, use of gonad shields.

The purpose of this study was to provide the required data enabling the determination of testicular dose and the assessment of the subsequent risk of infertility for individual patients undergoing radiotherapy for rectal carcinoma.

\section{Materials and methods}

Phantom measurements. Dose measurements were performed on an anthropomorphic phantom (Alderson, Research Labs, Stanford, CA), which has been broadly employed for measuring peripheral doses in radiation therapy (2,7-11). The Alderson Rando phantom is made of tissue equivalent material and it is transected at $2.5 \mathrm{~cm}$ intervals. It corresponds to a male human body with a height of $175 \mathrm{~cm}$ and weight of $73.5 \mathrm{~kg}$. A piece of perspex $3 \mathrm{~cm}$ in diameter and $5 \mathrm{~cm}$ long was placed between the legs of the phantom to simulate a human testicle. A hole was constructed to allow the placement of a thimble ionization chamber (M31002, PTW, Freiburg, Germany) at a point corresponding to the center of the testicle. The chamber was connected to an electrometer (UNIDOS, PTW). The ion chamber and the electrometer were traceable to the National Physical Laboratory. The uncertainty of ionization measurements has been estimated to be $0.5 \%$ (12). Phantom exposures were generated with a $6 \mathrm{MV} \mathrm{X}$-ray beam produced by a linear accelerator (Philips SL75/5, The Netherlands).

Radiotherapy for rectal cancer is currently performed using either a four-field box technique consisting of an anteroposterior (AP), a posteroanterior (PA) a right lateral (RL) and a left lateral (LL) portals or a three-beam box arrangement 
with a PA and a lateral parallel pair (1). A total tumour dose of $4500 \mathrm{cGy}$ is usually given in $180 \mathrm{cGy}$ daily fractions by equally weighted three or four field treatments (1). AP, PA and lateral field irradiations were simulated on the Rando phantom, which was in the prone position. Dose data were collected separately for each of the above fields. Lead blocks were used to shield radiosensitive structures in parts of AP, PA and lateral portals. The target isocenter was located in the midline of the phantom section no. 30 at a depth of $7 \mathrm{~cm}$ from the posterior phantom surface. The distance separating the field isocenter from the center of the artificial testicle was $14 \mathrm{~cm}$.

Testicular dose was measured for three different field sizes which can be applied during radiotherapy for rectal cancer. The dimensions of AP/PA fields were 10x14, 12.5x15.5 and $15 \times 17 \mathrm{~cm}^{2}$. The corresponding lateral field sizes were 9x14, $11.5 \times 15.5,14 \times 17 \mathrm{~cm}^{2}$, respectively. To provide dosimetric data for patients with different height from that of the Rando phantom, the effect of the distance between the testes and the primary irradiation field on the gonadal dose was examined. For all dimensions of AP, PA and lateral fields, testicular dose was measured at the following distances from the field isocenter: $11,12,13,15$ and $16 \mathrm{~cm}$. The above measurement points correspond to all possible positions of male gonads in respect to the irradiated area.

Effect of wedges on testicular dose. Additional measurements were performed to study the effect of wedge introduction into lateral beams on the testicular dose. The treatment unit of our department is equipped with a single motorized wedge filter with a stated angle of $60^{\circ}$. The wedged field can be combined with an open field to produce any effective wedge angle from $0^{\circ}$ to $60^{\circ}$ depending on the beam weight imposed on the wedged field. Based on the universal wedge equation presented by Zwicker et al (13) the weights of the wedged beams were selected to provide the following effective wedge angles: $30^{\circ}$, $45^{\circ}$ and $60^{\circ}$. Dose measurements were carried out for the above wedge angles using all lateral field sizes at a constant distance of $14 \mathrm{~cm}$ from the field isocenter. Correction factors representing the ratio of testicular dose from wedged lateral beams to that from open fields were calculated.

Effect of tissue thickness on testicular dose. The dependence of the testicular dose upon the thickness of the tissue irradiated by the primary beam was investigated. For AP and PA field irradiations, the phantom thickness at the entrance point of the beam was $20 \mathrm{~cm}$. Regarding the lateral irradiations, the corresponding thickness was $29 \mathrm{~cm}$. Testicular dose was measured using all AP, PA and lateral field sizes by adding various sheets of tissue-equivalent bolus material with thickness of 2, 4 and $6 \mathrm{~cm}$ to pelvic region in order to increase the size of the phantom. For each phantom thickness, a correction factor denoting the ratio of testicular dose measured with bolus to that measured without bolus was determined.

Testicular dose reduction. Conventional gonad shield based on the placement of a lead block on a shadow tray was initially employed to reduce testicular dose. The block was selected to have large dimensions (12 cm long, $12 \mathrm{~cm}$ wide, $8 \mathrm{~cm}$ thick) and it was abutted with the inferior border of

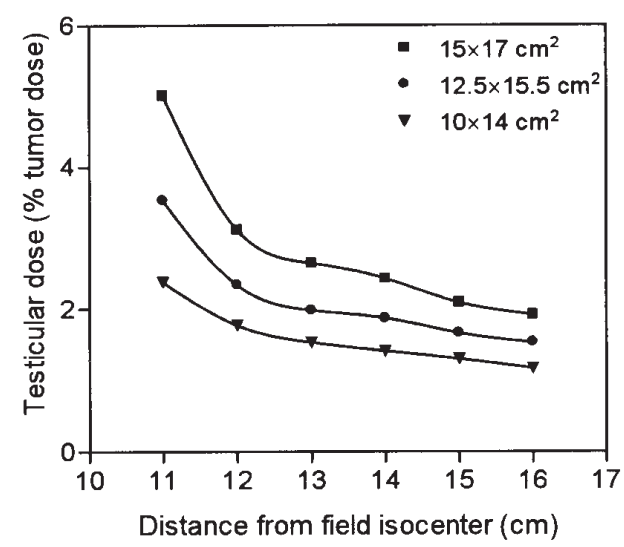

(a)

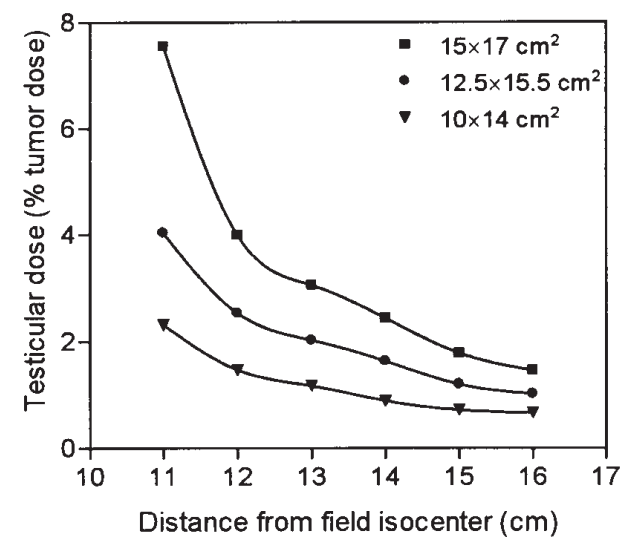

(b)

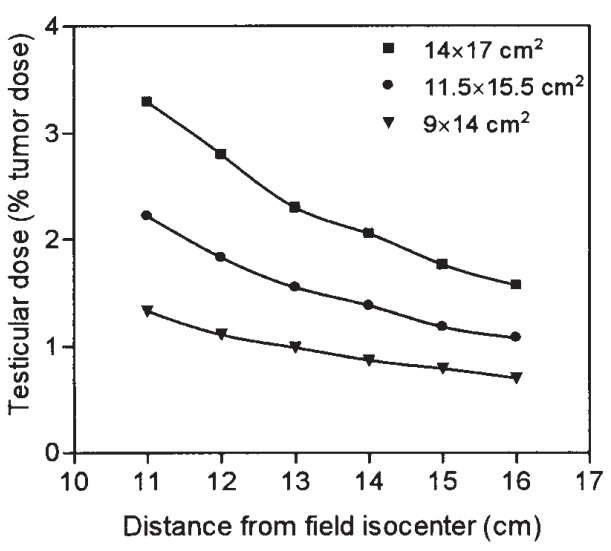

(c)

Figure 1. Testicular dose measurements resulting from (a) AP, (b) PA and (c) lateral field irradiations.

the treatment fields. Furthermore, a commercially available round shield (Model 37-937, Cardinal Health, OH) was used to protect the testes. The artificial testicle was placed between the top and bottom halves of the shield made of $1.27 \mathrm{~cm}$ thick lead. The shield's halves were joined together with rubber straps and they were lying on a groove. The height of the groove could be adjusted using a hand knob. For all dimensions of AP, PA and lateral fields, the absorbed dose to a shielded testicle was measured at a distance of $14 \mathrm{~cm}$ from the field center. 


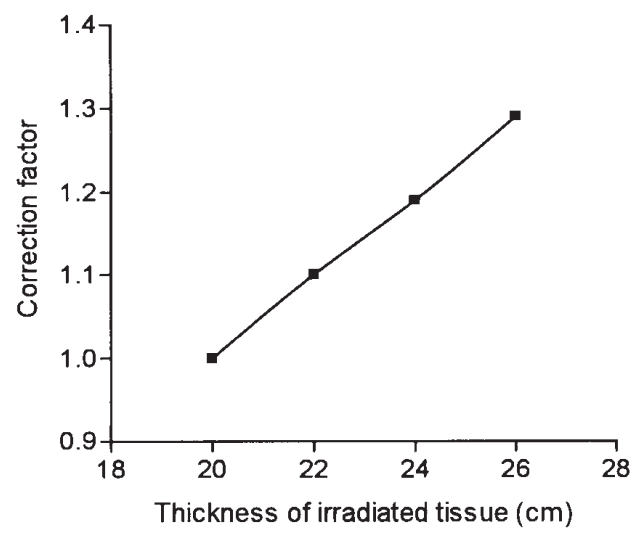

(a)

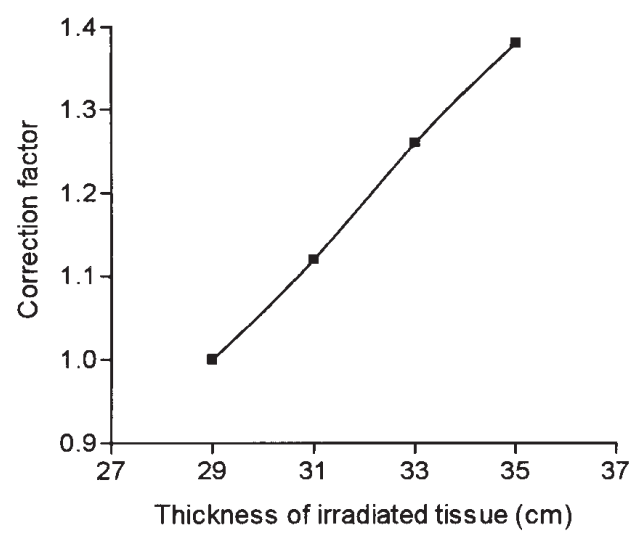

(b)

Figure 2. Correction factors (T) to estimate testicular dose for various tissue thicknesses irradiated from (a) AP and (b) lateral beams.

Patient measurements. The radiation dose received by the testes was measured in five patients undergoing radiotherapy for rectal cancer. In three cases, a four-field pelvic box technique was used, while two patients were irradiated by a three-field technique. Informed consent was obtained from all patients. Testicular dose was measured for the first day of the treatment. The prescribed daily dose was $180 \mathrm{cGy}$. To accomplish in vivo measurements, we asked the patients to remove their testes from the midline. Simulator films were used to verify that the artificial testicle equipped with the ion chamber was placed at the same distance from the lower field border with that of the human testicle. The measured gonadal dose was compared with the dose anticipated from the phantom measurements.

\section{Results}

Phantom measurements. Testicular dose measurements resulting from AP, PA and lateral field irradiations are shown in Fig. 1. Radiotherapy for rectal cancer using a three-field technique provided a total testicular dose of $0.7-4.7 \%$ of the given tumour dose depending upon the field dimensions applied and the distance from the primary irradiation field. The corresponding testicular dose from a four-field box technique was $0.8-4.8 \%$ of the prescribed target dose. Based
Table I. Correction factors (F) showing the ratio of testicular dose from wedged lateral beams to that from open beams.

\begin{tabular}{lccc}
\hline & \multicolumn{3}{c}{ Wedge angle } \\
\cline { 2 - 4 } Field size $\left(\mathrm{cm}^{2}\right)$ & $30^{\circ}$ & $45^{\circ}$ & $60^{\circ}$ \\
\hline $9 \times 14$ & 1.0 & 1.1 & 1.1 \\
$11.5 \times 15.5$ & 1.3 & 1.6 & 1.8 \\
$14 \times 17$ & 1.6 & 1.9 & 2.2 \\
\hline
\end{tabular}

Table II. Radiation dose to shielded testes.

\begin{tabular}{lccc}
\hline & & \multicolumn{2}{c}{ Testicular dose (\% tumour dose) } \\
\cline { 3 - 4 } Field & $\begin{array}{c}\text { Field size } \\
\left(\mathrm{cm}^{2}\right)\end{array}$ & $\begin{array}{c}\text { Round } \\
\text { shield }\end{array}$ & $\begin{array}{c}\text { Conventional } \\
\text { shield }\end{array}$ \\
\hline AP & $10 \times 14$ & 0.4 & 0.8 \\
& $12.5 \times 15.5$ & 0.6 & 1.2 \\
& $15 \times 17$ & 0.8 & 1.5 \\
PA & $10 \times 14$ & 0.3 & 0.6 \\
& $12.5 \times 15.5$ & 0.5 & 1.2 \\
& $15 \times 17$ & 0.8 & 1.8 \\
Lateral & $9 \times 14$ & 0.2 & 0.5 \\
& $11.5 \times 15.5$ & 0.5 & 1.1 \\
& $14 \times 17$ & 0.7 & 1.5 \\
\hline
\end{tabular}

on the above results, the total gonadal dose may vary from 32 to $216 \mathrm{cGy}$ for a treatment course giving $4500 \mathrm{cGy}$ to the tumour site.

The dependence of the testicular dose upon the tissue thickness along the beam axis is presented in Fig. 2. For each phantom thickness, the correction factor (T) in the above figure corresponds to the mean value of the three factors obtained from exposures with the three different field sizes. For lateral field irradiations, the testicular dose increased by a factor up to 1.4 depending upon the thickness of the tissue irradiated by the beam. Regarding the AP treatment fields, the corresponding factor was up to 1.3. The increase of tissue thickness had no effect on the testicular dose from PA field irradiations. Phantom exposures with PA fields showed that the absolute difference between the gonadal dose measured for the standard thickness of $20 \mathrm{~cm}$ with those obtained for greater girths was always $<8.5 \%$. Testicular dose from wedged lateral beams was up to 2.2 times higher than that from open fields. The correction factors (F) associated with the increase of testicular dose for three different wedge angles and field sizes are presented in Table I.

The radiation doses to testes protected by the two different gonad shields are shown in Table II. The mean ratio of testicular dose using the conventional shield to that measured using the round shield was 2.1. For a three- or a four-field 
irradiation technique, the use of lead blocks placed on a shadow tray reduced the total testicular dose by $27-41 \%$ depending upon the field size, in comparison with the dose to unshielded testes determined from Fig. 1. The corresponding total testicular dose reduction using the round gonad shield was $66-74 \%$.

The radiation dose to testes resulting from radiotherapy for rectal cancer can be calculated using the following equation:

$$
\mathrm{D}=\sum_{\mathrm{i}=1}^{\mathrm{n}} \mathrm{w}_{\mathrm{i}}\left(\mathrm{D}_{\mathrm{r}, \mathrm{d}}\right)_{\mathrm{i}} \mathrm{F}_{\mathrm{i}} \mathrm{T}_{\mathrm{i}}
$$

where $\mathrm{D}$ is the testicular dose expressed as a percentage of the given tumour dose, $\mathrm{i}$ is the irradiation field, $\mathrm{n}$ equals to 3 or 4 depending on the irradiation technique employed, $w$ is the beam weight which varies from 0 to $1, \mathrm{D}_{\mathrm{r}, \mathrm{d}}$ is the testicular dose as a function of field size ( $r$ ) and distance from field isocenter (d) provided by Fig. 1, F is the correction factor applied during lateral exposures with wedge filters and $\mathrm{T}$ is the correction factor used whenever the patient thickness along beam axis differs from that of the humanoid phantom.

Patient measurements. The mean value of the measured testicular doses was 7.6 cGy with a variation of 4.3-11.3 cGy for a single fraction of $180 \mathrm{cGy}$. The respective gonadal doses calculated using Equation i) ranged from 4.2 to $11.9 \mathrm{cGy}$ with a mean dose of $7.5 \mathrm{cGy}$. For one patient irradiated with field sizes larger than those used in the current study, the $D_{r, d}$ values inserted in Equation i) corresponded to the maximum doses presented in Fig. 1 for the specific distance separating the patient's testes from the field center. For the remaining patients, $D_{r, d}$ values were determined either directly from Fig. 1 or by interpolating the dose data of the above figure. The mean difference between the measured testicular doses and the respective calculated doses was $5.8 \%$ with a range of $1.0-9.6 \%$.

\section{Discussion}

Radiotherapy-induced damage of male gonadal function is dose-dependent. Previous studies have suggested that permanent infertility appears after a fractionated dose to testes of $200 \mathrm{cGy}(14,15)$. Greiner has reported that lower gonadal doses of $150 \mathrm{cGy}$ can also lead to permanent azoospermia (16). A partial recovery of sperm cell production has been observed in patients receiving testicular doses of 100-150 cGy $(16,17)$. Testicular exposure with fractionated doses $<100$ cGy allows a complete recovery of spermatogenesis $(15,16)$. Phantom and patient measurements presented here revealed that testicular dose can exceed the threshold value of $100 \mathrm{cGy}$ depending upon the field arrangement used, field dimensions, distance from irradiated area, introduction of wedges and tumour dose.

The current study provides a simple method to estimate the testicular dose associated with radiotherapy for rectal cancer using a three or four-field irradiation technique with any beam weight. Moreover, testicular dose can be estimated during irradiation with opposing AP and PA fields, which may be applied during treatment for carcinoma of the rectum (18). The dependence of testicular dose upon the patient height and patient thickness irradiated by the primary beam was taken into account. Therefore, the method presented here can be applied for individual patients with different somatometric characteristics from those of the anthropomorphic phantom used. The good agreement between the measured and the calculated gonadal doses gives a strong evidence about the reliability and accuracy of the proposed method.

Limited information has been reported about gonadal dose reduction during radiotherapy for rectal cancer. Hollenhorst et al (6) reported a total testicular dose of $75 \mathrm{cGy}$ for a single patient treated for carcinoma of the rectum with a scrotal block consisting of an alloy of lead, bismuth and zinc. No data were given about the efficiency of the specific gonad shield. In the current study, conventional shield based on the placement of lead blocks at a distance from the pelvis reduced the testicular dose by $<41 \%$ compared with the unshielded setup. The use of a round shield, which can enclose the testes at the time of irradiation, was much more effective since it reduced gonadal dose by $>66 \%$. Our dosimetric results clearly indicate that significant testicular dose reductions can be achieved only when shields specially constructed for gonadal protection are placed between the thighs at the region of the scrotum. Gonadal doses as low as 10 cGy can induce temporary changes to spermatogonia (15). Therefore, gonad shields should be applied in all male patients for whom fertility preservation is a concern.

Although the positioning of appropriate shield can be considered as the primary choice for gonadal protection during radiation therapy, simple modifications of treatment parameters can also result in testicular dose reduction. For lateral field irradiations, it is advantageous to use a wedge angle as small as possible whenever the wedge introduction is unavoidable. Based on our dosimetric results, radiotherapy for obese patients can be performed with the three-field instead of the four-field technique because of the increase of testicular dose with tissue thickness irradiated by an AP beam. Special consideration should also be given by radiotherapists during treatment planning procedure to apply the absolutely necessary field margins due to the gonadal dose reduction associated with the restriction of the irradiated area.

Sources of error in testicular dose estimation are mainly related to the uncertainty of the ionization measurements. A limitation of this study might be the measurement of gonadal dose at a single point corresponding to the center of the human testicle. However, a previous publication concerning radiotherapy for prostate cancer has reported a small variation of absorbed dose across the testicle (19). All phantom exposures of this study were generated using a linear accelerator operating at $6 \mathrm{MV}$. We consider that the method presented here can be applied for individual patients treated with different X-ray megavoltage beams based on the limited dependence of peripheral doses upon the photon energy $(20,21)$.

In conclusion, radiotherapy for rectal cancer may be associated with the induction of permanent or temporary reduced male fertility potential. The use of purpose built gonad shields should be considered as necessary for male patients who may wish to father children. The current study provides an easily applicable method enabling the calculation 
of the absorbed dose to testes. The procedure of testicular dose estimation before treatment course gives the possibility to radiotherapists to initially assess the risk of infertility and, then, to accomplish all feasible modifications in treatment parameters to reduce the above risk.

\section{References}

1. Myerson RJ: Colon and rectum. In: Principles and Practice of Radiation Oncology. Perez CA, Brady LW, Halperin EC and Schmidt-Ullrich RK (eds). Lippincott Williams \& Wilkins, Philadelphia, pp1607-1629, 2004.

2. Budgell GJ, Cowan RA and Hounsell AR: Prediction of scattered dose to the testes in abdominopelvic radiotherapy. Clin Oncol 13: $120-125,2001$.

3. Piroth MD, Hensley F, Wannenmacher M and Zierhut D: Male gonadal dose in adjuvant 3-d pelvic irradiation after anterior resection of rectal cancer. Influence to fertility. Strahlenther Onkol 179: 754-759, 2003.

4. Hall EJ (ed): Radiobiology for the Radiologist. 5th edition. Lippincott Williams \& Wilkins, Philadelphia, 2000.

5. Dueland S, Guren MG, Olsen DR, Poulsen JP and Tveit KM: Radiation therapy induced changes in male sex hormone levels in rectal cancer patients. Radiother Oncol 68: 249-253, 2003.

6. Hollenhorst H, Schaffer M, Romano M, Reiner M, Siefert A, Schaffer P, Quanz A and Duhmke E: Optimized radiation of pelvic volumes in the clinical setting by using a novel belly-board with integrated gonadal shielding. Med Dosim 29: 173-178, 2004.

7. Jacobsen KD, Olsen DR, Fossa K and Fossa SD: External beam abdominal radiotherapy in patients with seminoma stage I: field type, testicular dose, and spermatogenesis. Int J Radiat Oncol Biol Phys 38: 95-102, 1997.

8. Mazonakis M, Damilakis J, Theoharopoulos N, Varveris H and Gourtsoyiannis N: Brain radiotherapy during pregnancy: an analysis of conceptus dose using anthropomorphic phantoms. Br J Radiol 72: 274-278, 1999.

9. Mazonakis M, Varveris H, Damilakis J, Theoharopoulos $\mathrm{N}$ and Gourtsoyiannis N: Radiation dose to conceptus resulting from tangential breast irradiation. Int J Radiat Oncol Biol Phys 55: 386-391, 2003.
10. Mazonakis M, Damilakis J, Varveris H, Fasoulaki M and Gourtsoyiannis N: Risk estimation of radiation-induced thyroid cancer from treatment of brain tumors in adults and children. Int J Oncol 22: 221-225, 2003.

11. Mazonakis M, Damilakis J, Varveris H, and Gourtsoyiannis N: Therapeutic external irradiation in women of reproductive age: risk estimation of hereditary effects. Br J Radiol 77: 847-850, 2004.

12. Tzedakis A, Damilakis JE, Mazonakis M, Stratakis J, Varveris H and Gourtsoyiannis N: Influence of initial electron beam parameters on Monte Carlo calculated absorbed dose distributions for radiotherapy photon beams. Med Phys 31: 907-913, 2004.

13. Zwicker RD, Shahabi S, Wu A and Sternick ES: Effective wedge angles for 6-MV wedges. Med Phys 12: 347-349, 1985.

14. Ash P: The influence of radiation on fertility in man. Br J Radiol 53: 271-278, 1980.

15. Howell S and Shalet S: Gonadal damage from chemotherapy and radiotherapy. Endocrinol Metab Clin North Am 27: 927-943, 1998.

16. Greiner R: Spermatogenesis after fractionated, low-dose irradiation of the gonads. Strahlentherapie 158: 342-355, 1982.

17. Hansen PV, Trykker H, Svennekjaer IL and Hvolby J: Longterm recovery of spermatogenesis after radiotherapy in patients with testicular cancer. Radiother Oncol 18: 117-125, 1990.

18. Frykholm GJ, Isacsson U, Nygard K, Montelius A, Jung B, Pahlman L and Glimelius B: Preoperative radiotherapy in rectal carcinoma-aspects of acute adverse effects and radiation technique. Int J Radiat Oncol Biol Phys 35: 1039-1048, 1996.

19. Amies CJ, Mameghan H, Rose A and Fisher RJ: Testicular doses in definitive radiation therapy for localized prostate cancer. Int J Radiat Oncol Biol Phys 32: 839-846, 1995.

20. Stovall M, Blackwell CR, Cundiff J, Novack DH, Palta JR, Wagner LK, Webster EW and Shalek RJ: Fetal dose from radiotherapy with photon beams: report of AAPM Radiation Therapy Committee Task Group no. 36. Med Phys 22: 63-82, 1995.

21. Van der Giessen PH: A simple and generally applicable method to estimate the peripheral dose in radiation teletherapy with high energy X-rays or gamma radiation. Int J Radiat Oncol Biol Phys 35: 1059-1068, 1996. 